\title{
A!
}

This is an electronic reprint of the original article.

This reprint may differ from the original in pagination and typographic detail.

Maisi, Ville; Saira, O.-P.; Pashkin, Yu.A.; Tsai, J.S.; Averin, D.V.; Pekola, J.P.

\section{Real-Time Observation of Discrete Andreev Tunneling Events}

Published in:

Physical Review Letters

DOI:

10.1103/PhysRevLett.106.217003

Published: 25/05/2011

Document Version

Publisher's PDF, also known as Version of record

Please cite the original version:

Maisi, V., Saira, O-P., Pashkin, Y. A., Tsai, J. S., Averin, D. V., \& Pekola, J. P. (2011). Real-Time Observation of Discrete Andreev Tunneling Events. Physical Review Letters, 106(21), 1-4. [217003].

https://doi.org/10.1103/PhysRevLett.106.217003

This material is protected by copyright and other intellectual property rights, and duplication or sale of all or part of any of the repository collections is not permitted, except that material may be duplicated by you for your research use or educational purposes in electronic or print form. You must obtain permission for any other use. Electronic or print copies may not be offered, whether for sale or otherwise to anyone who is not an authorised user. 


\title{
Real-Time Observation of Discrete Andreev Tunneling Events
}

\author{
V.F. Maisi, ${ }^{1,2, *}$ O.-P. Saira, ${ }^{1}$ Yu. A. Pashkin,,${ }^{3, \dagger}$ J. S. Tsai, ${ }^{3}$ D. V. Averin,${ }^{4}$ and J. P. Pekola ${ }^{1}$ \\ ${ }^{1}$ Low Temperature Laboratory, Aalto University, P.O. Box 13500, 00076 Aalto, Finland \\ ${ }^{2}$ Centre for Metrology and Accreditation (MIKES), P.O. Box 9, 02151 Espoo, Finland \\ ${ }^{3}$ NEC Green Innovation Research Laboratories and RIKEN Advanced Science Institute, \\ 34 Miyukigaoka, Tsukuba, Ibaraki 305-8501, Japan \\ ${ }^{4}$ Department of Physics and Astronomy, Stony Brook University, SUNY, Stony Brook, New York 11794-3800, USA
}

(Received 28 February 2011; published 25 May 2011)

\begin{abstract}
We provide a direct proof of two-electron Andreev transitions in a superconductor-normal-metal tunnel junction by detecting them in a real-time electron counting experiment. Our results are consistent with ballistic Andreev transport with an order of magnitude higher rate than expected for a uniform barrier, suggesting that only part of the interface is effectively contributing to the transport. These findings are quantitatively supported by our direct current measurements in single-electron transistors with similar tunnel barriers.
\end{abstract}

DOI: 10.1103/PhysRevLett.106.217003

PACS numbers: 74.50.+r, 73.23.-b, 73.40.Gk

Electronic transport across a boundary between conductors with dissimilar carriers is a nontrivial process. Of particular interest in this respect is the transport through a superconductor-normal-metal interface that at low energies is dominated by Andreev reflection [1-10], where a Cooper pair in a superconductor is converted into two electrons in the normal metal or vice versa. Here we employ electron counting techniques [11-17] to detect these Andreev events. Since the observed rate depends on the coherence of the two electrons involved in the transition, we obtain, as a result, a fingerprint of the junction electrodes and the tunnel barrier.

The techniques used for observing individual electrons are based on the Coulomb blockade effect where the electrostatic energy of a small metallic island changes noticeably when only one elementary charge $e$ is placed on or removed from it. In the present experiment, we employ an isolated single-electron box where a superconducting island is connected to a normal metal one [17], but neither of these two is connected galvanically to the external circuitry. The electron tunneling rates between the islands are then sufficiently low to be monitored by lowfrequency electrometry and are described in detail by relatively simple theoretical considerations $[17,18]$. We use a single-electron transistor (SET) [11-15,17,19-22] as an ultrasensitive electrometer. With charge sensitivity as good as $10^{-5} e / \sqrt{\mathrm{Hz}}[22-24]$, it is capable to detect individual electrons with high precision. In Fig. 1, we show a micrograph of our sample fabricated by standard $e$-beam processing.

The tunneling rates and resulting charge distribution between the two islands of the isolated box can be adjusted with an offset charge induced by a gate voltage. The electrostatic energy of a state with $n$ excess electrons on one of the islands is given by $E_{n}=E_{c}\left(n-n_{g}\right)^{2}$, where $E_{c}$ is the charging energy for individual electrons and $n_{g}$ is the normalized offset charge that can be viewed as the polarization charge on the gate capacitor and determines the energetically preferred way to occupy the different charge states $n$ [20]. In Fig. 2(a), the two extreme cases are shown. In the Coulomb blockade regime for single electrons, $n_{g}$ is an integer and the state $n=n_{g}$ has the minimal energy $E_{n}=0$. To enter an excited state, one electron can tunnel either into or out of the island [dotted black lines with arrows in Fig. 2(a)], but energy $E_{c}$ has to be provided for the tunneling electron in addition to the Cooper pair breaking energy equal to or larger than the superconducting energy gap $\Delta$ [25]. In the other extreme, at degeneracy with half-integer $n_{g}$, two electron states differing by charge $e$ have equal minimal energy and hence are equally populated. The tunneling rate between them is higher than in the Coulomb blockade regime as no extra energy for charging is needed. For Andreev reflection [solid red lines with

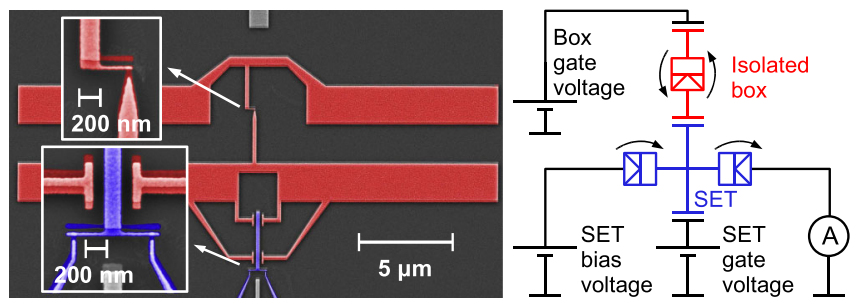

FIG. 1 (color online). Scanning electron micrograph of the measured structure and the schematic layout of the measurement setup. The isolated electron box consists of two metallic islands, seen as $25 \mu \mathrm{m}$ long rectangles (colored red). They are connected to each other by a normal-metal-insulator-superconductor tunnel junction. Tunneling of electrons through the junction is monitored with a dc SET electrometer (in blue) coupled capacitively to one of the box islands. The normal-metal-insulatorsuperconductor junction (top) and detector (bottom) are shown magnified on the left side of the main micrograph. 
(a)

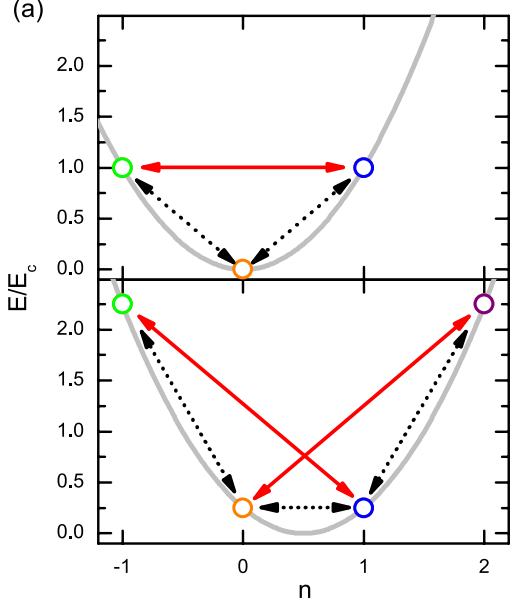

(b)

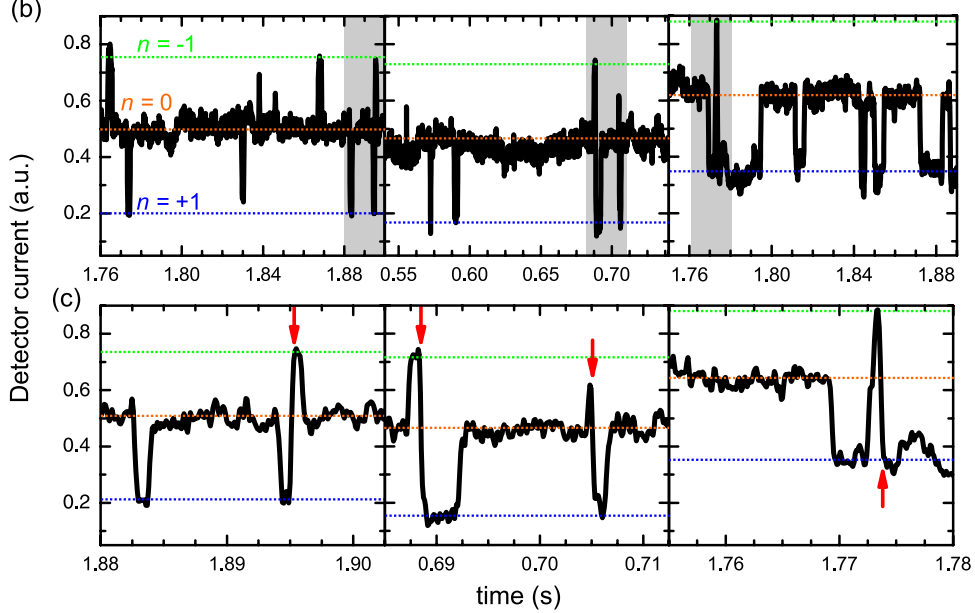

FIG. 2 (color online). Energy levels of the various charge states and typical observed time traces of the current through the detector. (a) Low lying levels of the box in the Coulomb blockade (upper panel) and at degeneracy (lower panel). Dotted (black) and solid (red) arrows indicate one- and two-electron processes, respectively. (b) Measured time traces of the detector current showing the charge state of the electron box as a function of time. The leftmost panel presents the case of Coulomb blockade. The rightmost panel depicts the opposite limit where the two charge states are equal in energy (degeneracy). The trace in the center is taken halfway between these two cases. (c) Gray sections of the traces of (b) zoomed. Vertical arrows indicate two-electron events.

arrows in Fig. 2(a)], the energy cost of charging is calculated similarly, but now the initial and final states are separated by two electrons, and the energy cost of breaking a Cooper pair is avoided since complete pairs tunnel at once.

In the experiment (see the supplemental material for additional details [26]), we measured time traces of the detector current at various biasing conditions of the box. With all the other parameters fixed, the detector gate voltage was adjusted to maximize charge sensitivity and dynamic range. The observed current jumps [Figs. 2(b) and $2(\mathrm{c})]$ are attributed to the tunneling events between the two islands. The switching rate depends on the gate voltage of the box and hence on its charge state, being lowest in the Coulomb blockade regime (leftmost panel) and highest at degeneracy (rightmost panel). The events observed in the traces [gray regions of Fig. 2(b) zoomed in 2(c)] indicate that individual electrons tunnel between the islands: In the Coulomb blockade regime they hop rarely from the lowest $(n=0)$ to the higher $(n= \pm 1)$ energy states and back, while at degeneracy the electrons tunnel frequently between the two lowest states and only occasionally the system enters a higher lying level. More interestingly, the traces also show the coincident events, pointed out by the vertical arrows, where two electrons appear to tunnel simultaneously. In the following, we show that most of these events represent Andreev tunneling.

Because of the finite measurement bandwidth, limited to $1 \mathrm{kHz}$ by the dc readout of the electrometer, events resembling two-electron Andreev tunneling could in principle arise from almost coincidental tunneling of two independent quasiparticles. To assess this option, we recorded time traces for several minutes at each gate offset value. From the traces we determined the distribution of the time $t$ spent in the state $n=0$ before a transition took place. In Fig. 3(a), we show such a distribution on the left for Coulomb blockade $\left(n_{g}=0\right)$, in the center for $n_{g}=0.25$, and on the right for near degeneracy $\left(n_{g}=0.45\right)$. A direct transition (Andreev tunneling) between states $n= \pm 1$ contributes here as essentially a $t=0$ event since the time separation between the two electrons tunneling in the Andreev process should be on the order of $\hbar / \Delta$, which is many orders of magnitude smaller than the time scales relevant in Fig. 3(a). Overall, the distribution is exponential as we have Poisson distributed one-electron tunneling processes. However, at small lifetimes in the Coulomb blockade regime, the data point indicated by the horizontal arrow does not follow the exponential dependence and corresponds to excessively many events. This clear separation of the short-lifetime events from the one-electron transitions shows that the majority of these events are not coincidental one-electron tunneling but rather two electrons tunneling concurrently. When the box offset charge is adjusted closer to degeneracy, the anomalous data point gradually merges to the rest of the data, in accordance with its interpretation in terms of Andreev transitions, since the energy cost of the two-electron tunneling increases. We emphasize that the charging energy should be small $\left(E_{c}<\Delta\right)$ for Andreev tunneling to occur, since for large $E_{c}$, it is not energetically favorable [17].

For quantitative analysis, we counted the number of events $N_{j}$ for each possible forward and backward oneand two-electron tunneling process for each initial charge state $n$. The corresponding tunneling rate was then obtained as $\Gamma_{j}=N_{j} /\left(\langle t\rangle N_{\Sigma}\right)$, where $\langle t\rangle$ is the average lifetime of the initial state $n$ and $N_{\Sigma}$ the total number of all transitions out of this state. The denominator $\langle t\rangle N_{\Sigma}$ therefore corresponds to the total time spent in the initial state. 
(a)

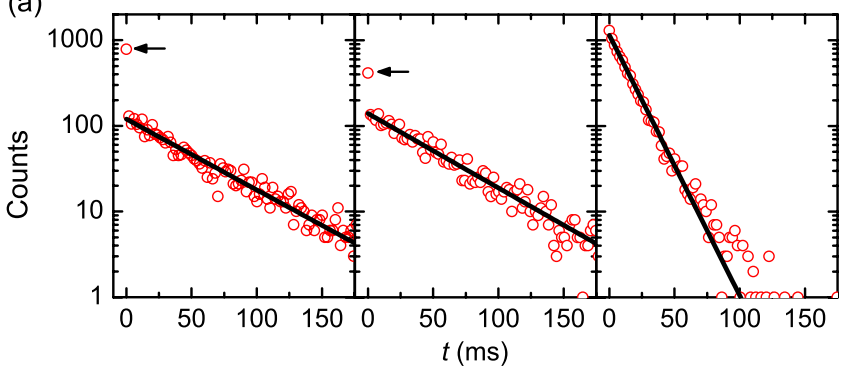

(b)

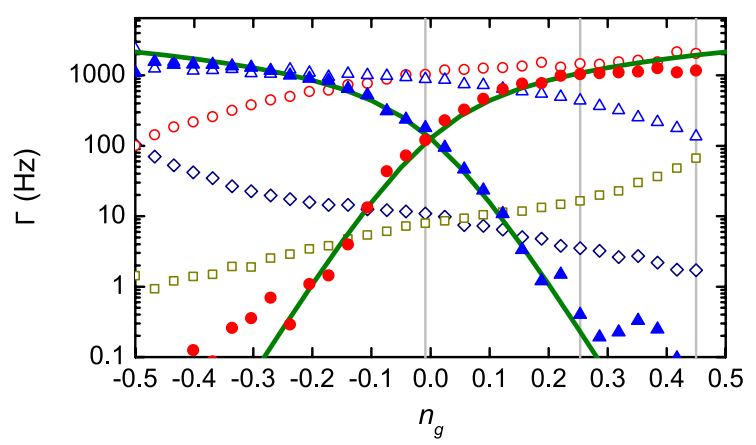

FIG. 3 (color online). Lifetime distributions and tunneling rates. (a) Lifetime distributions of the $n=0$ charge state in the Coulomb blockade, at an intermediate gate position, and close to degeneracy, from left to right. The corresponding gate positions are indicated by vertical lines in (b). Excessively many events at small times are pointed out by horizontal arrows. (b) One-electron (open symbols) and two-electron (solid symbols) tunneling rates estimated from the counted events at the base temperature. Red circles and blue triangles denote the tunneling rates out of states $n=-1$ and 1 , respectively. Dark blue diamonds and dark yellow squares denote tunneling rates out of state 0 to states -1 and 1 , respectively. Dark green solid lines present calculated Andreev rates with $\zeta=4 \times 10^{-5}$.

Transitions with the charge number changing by $\Delta n= \pm 2$ and occurring within $0.4 \mathrm{~ms}$ were interpreted as twoelectron tunneling and all other events as one-electron tunneling. The time window was chosen such that it essentially captures all the excess events shown in the histograms of Fig. 3(a) but minimizes the number of coincidental one-electron events. The results for both processes at the temperature of $60 \mathrm{mK}$ are shown in Fig. 3(b). The parameter values which determine the first-order tunneling rates in the box junction, the tunneling resistance $R_{T}=2 \mathrm{M} \Omega$, the superconducting energy gap $\Delta=$ $216 \mu \mathrm{eV}$, and the charging energy $E_{c}=0.2 \Delta$, were determined from the one-electron tunneling rates measured at temperatures ranging from 60 to $200 \mathrm{mK}$ as in Ref. [17].

For analyzing the Andreev tunneling rates quantitatively, we assume a ballistic model of the tunnel barrier. Then, the rate is given by Eq. (3) of Ref. [27]. Apart from the parameter values obtained from one-electron tunneling, the only parameter to be determined for the two-electron tunneling is its overall magnitude controlled by $\zeta \equiv$ $\left(\hbar / R_{T} e^{2}\right) / \mathcal{N}$, where $\mathcal{N}$ is the effective number of the conduction channels in the junction, $\mathcal{N}=A / A_{\mathrm{ch}}$. Here $A_{\text {ch }}$ is the area of one conduction channel and
$A=40 \mathrm{~nm} \times 35 \mathrm{~nm}$ the junction area estimated from the scanning electron micrograph. Ideally, for our aluminum oxide tunnel barriers one should have $A_{\mathrm{ch}} \simeq 2 \mathrm{~nm}^{2}$; see the supplemental material [26]. However, to fit the observed tunneling rates, we must adopt an order of magnitude higher value, $A_{\mathrm{ch}}=30 \mathrm{~nm}^{2}$, leading to an order of magnitude higher rates. As a result, we obtain the green lines of Fig. 3(b) for forward and backward tunneling, demonstrating a good agreement between theory and experiments at different charging energy costs tuned by $n_{g}$.

An order of magnitude larger area of a conduction channel is in line with independently performed SET measurements described in detail below and with the conclusions in, e.g., Refs. [6,10], for larger tunnel junctions that exhibit diffusive Andreev tunneling. We attribute this to the imperfections of the barrier, with only a small portion of the junction area dominating the transport. This fact can be understood by noting that the standard parameters of the aluminum oxide tunnel barriers [26,28,29] imply that, for example, variation of the barrier thickness by one atomic layer (i.e., by about $0.3 \mathrm{~nm}$ ) results in the specific conductance change by about a factor of 10 . This means that even relatively small fluctuations of the barrier thickness of the magnitude of one to two atomic layers in comparison with the average barrier thickness of 6 atomic layers can reduce the effective area of the region dominating the barrier transparency to about $10 \%$ of the total junction area.

As an additional way of studying the two-electron tunneling, we measured current-voltage characteristics of basic SET structures with superconducting leads and a normal metal island. A micrograph of one of the four devices is shown in the inset in Fig. 4. All the SETs were fabricated in the same batch so that the junction area $\left(E_{c}\right)$ varies with constant $\zeta \propto\left(A R_{T}\right)^{-1}$. Here Andreev tunneling has to be extracted from the measured data containing a contribution also from one-electron processes. Each of the SETs was biased at voltage $V$, and the current $I$ flowing through the device was measured. At each bias voltage, the gate offset of the island was varied so that the maximal and minimal currents, corresponding to degeneracy and Coulomb blockade, respectively, were observed. The measured current values between these extremes are shown as the colored regions in Fig. 4 for the four devices having different $E_{c}$. The parameter values for $E_{c}, R_{T}$, and $\Delta$ were determined from large scale data dominated by oneelectron tunneling. Insets show the data for samples with the highest and lowest $E_{c}$. In the subgap region $e V<2 \Delta$, we have no current flowing through the highest charging energy sample (top) apart from the thermally activated single-electron tunneling. With smaller $E_{c}$, the onset of the Andreev current penetrates deeper into the subgap region with the threshold at $e V=2 E_{c}$, seen both in the experimental data and in the fits including Andreev processes (solid black lines), as the energy cost for two electrons tunneling into the island is lowered. With high 


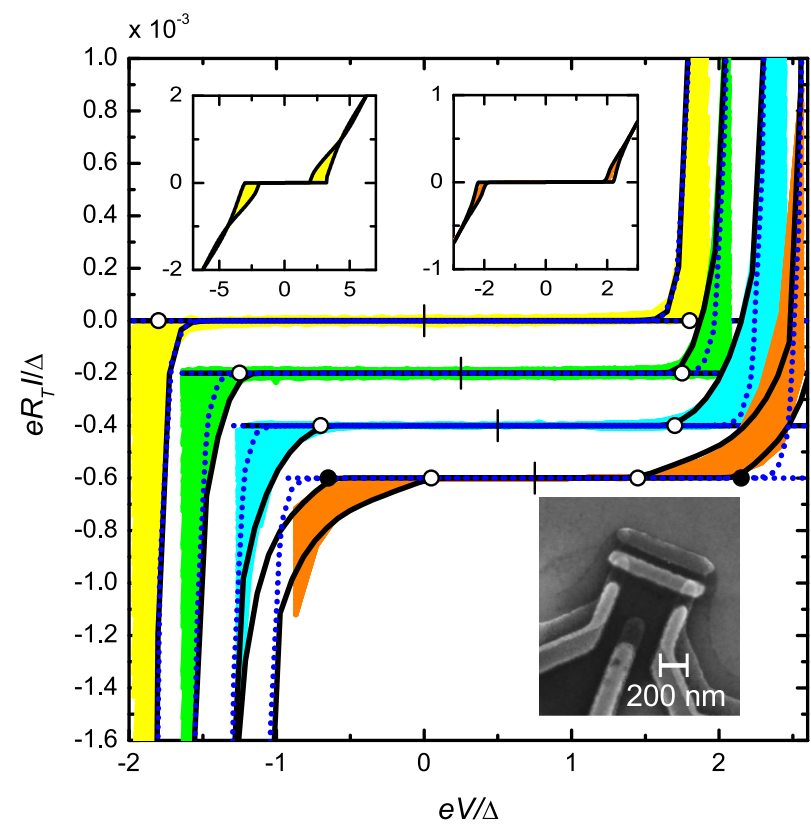

FIG. 4 (color online). Detection of Andreev current in SETs. Colored regions show measured subgap current for devices with charging energies $E_{c} / k_{B}=2.3,1.9,1.5$, and $0.86 \mathrm{~K}$ from top (yellow) to bottom (orange) at all gate offset values. The insets depict the larger scale measurements for samples with the highest and lowest charging energies and a scanning electron micrograph of one of the measured SETs. Solid black lines are fitted theoretical curves at degeneracy (maximal current) and in Coulomb blockade (minimal current) when Andreev tunneling is taken into account. Dotted blue lines present fits excluding Andreev processes. Tunneling resistances of the samples were $R_{T}=129,78,55$, and $31 \mathrm{k} \Omega$ in order of decreasing $E_{c}$, and superconducting gap $\Delta=216 \mu \mathrm{eV}$ for all of them. Open (solid) circles present the expected thresholds $e V= \pm 2 E_{c}$ $\left(e V= \pm 4 E_{c}\right)$ for Andreev tunneling at degeneracy (Coulomb blockade).

charging energies $E_{c} \gtrless \Delta$, it is sufficient to take into account only one-electron tunneling in the fits (dotted blue lines). In the numerical simulations for all the samples, we obtain as the only fit parameter for the subgap currents $\zeta \approx 4 \times 10^{-4}$, yielding the area of one conduction channel to be $A_{\mathrm{ch}}=30 \mathrm{~nm}^{2}$. This value was adopted to the analysis of the counting data above. Note that to increase the measured signal the SET junctions were oxidized less to have larger specific conductance $(\zeta)$ than the box junction.

In conclusion, we have detected tunneling in the time domain, allowing us to distinguish two-electron Andreev transitions from the usual one-electron processes unambiguously and on the level of individual events. This technique, unlike the direct current measurements, addresses the transition rates in the two directions separately, allowing for the study of the bias dependence of these rates also in the regime where currents are far too low to be detected by standard current measurements. The counting experiments disclose the detailed statistics of the two processes, and the results are consistent with the direct current measurements performed: As a technological result, both measurements indicate that the tunnel barriers are nonuniform.

The work has been supported partially by the Academy of Finland, Technology Industries of Finland Centennial Foundation, Väisälä Foundation, the Finnish National Graduate School in Nanoscience, MEXT kakenhi "Quantum Cybernetics," the JSPS through its FIRST Program, and the European Community's Seventh Framework Program under Grant Agreement No. 218783 (SCOPE). D. V. A. was supported in part by IARPA.

*ville.maisi@mikes.fi

†n leave from Lebedev Physical Institute, Moscow 119991, Russia.

[1] A. F. Andreev, Zh. Eksp. Teor. Fiz. 46, 1823 (1964) [Sov. Phys. JETP 19, 1228 (1964)].

[2] G.E. Blonder, M. Tinkham, and T. M. Klapwijk, Phys. Rev. B 25, 4515 (1982).

[3] T. M. Eiles, J. M. Martinis, and M. H. Devoret, Phys. Rev. Lett. 70, 1862 (1993).

[4] P. Lafarge et al., Nature (London) 365, 422 (1993).

[5] F. W. J. Hekking and Y. V. Nazarov, Phys. Rev. B 49, 6847 (1994).

[6] H. Pothier et al., Phys. Rev. Lett. 73, 2488 (1994).

[7] S. Rajauria et al., Phys. Rev. Lett. 100, 207002 (2008).

[8] A. S. Vasenko et al., Phys. Rev. B 81, 094513 (2010).

[9] J. Wei and V. Chandrasekhar, Nature Phys. 6, 494 (2010).

[10] T. Greibe et al., Phys. Rev. Lett. 106, 097001 (2011).

[11] J. M. Martinis, M. Nahum, and H. D. Jensen, Phys. Rev. Lett. 72, 904 (1994).

[12] P. D. Dresselhaus et al., Phys. Rev. Lett. 72, 3226 (1994).

[13] S. V. Lotkhov et al., Appl. Phys. Lett. 75, 2665 (1999).

[14] W. Lu et al., Nature (London) 423, 422 (2003).

[15] J. Bylander, T. Duty, and P. Delsing, Nature (London) 434, 361 (2005).

[16] T. Fujisawa et al., Science 312, 1634 (2006).

[17] O.-P. Saira et al., Phys. Rev. B 82, 155443 (2010).

[18] J. P. Pekola et al., Phys. Rev. Lett. 105, 026803 (2010).

[19] P. Lafarge et al., Z. Phys. B 85, 327 (1991).

[20] D. V. Averin and K. K. Likharev, J. Low Temp. Phys. 62, 345 (1986)

[21] T. A. Fulton and G. J. Dolan, Phys. Rev. Lett. 59, 109 (1987).

[22] G. Zimmerli et al., Appl. Phys. Lett. 61, 237 (1992).

[23] V. A. Krupenin et al., J. Appl. Phys. 84, 3212 (1998).

[24] L. Roschier et al., Appl. Phys. Lett. 78, 3295 (2001).

[25] I. Giaever, Phys. Rev. Lett. 5, 147 (1960).

[26] See supplemental material at http://link.aps.org/ supplemental/10.1103/PhysRevLett.106.217003 for details about sample fabrication, measurements, data analysis, and channel size estimate.

[27] D. V. Averin and J. P. Pekola, Phys. Rev. Lett. 101, 066801 (2008).

[28] Z. Tan et al., Appl. Phys. Lett. 93, 242109 (2008).

[29] M. Prunnila et al., J. Vac. Sci. Technol. B 28, 1026 (2010). 